Nortes Martínez-Artero, Rosa; Nortes Checa, Andrés (2016). Resolución de problemas, errores y dificultades en el grado de maestro de primaria. Revista de Investigación Educativa, 34(1), 103-117.

DOI: http://dx.doi.org/10.6018/rie.34.1.229501

\title{
Resolución de problemas, errores y dificultades en el grado de maestro de primaria
}

\author{
Problem solving, errors and difficulties \\ in the Primary School Degree
}

\author{
Rosa Nortes Martínez-Artero \\ Andrés Nortes Checa \\ Departamento de Didáctica de las Ciencias Matemáticas y Sociales \\ Universidad de Murcia
}

\begin{abstract}
Resumen
Este artículo aborda errores y dificultades que tienen los futuros maestros al resolver problemas elementales de Matemáticas. Se ha utilizado la prueba de Matemáticas para el ingreso en el cuerpo de maestros de la comunidad de Madrid, un cuestionario de actitud y otro de ansiedad aplicados a 142 alumnos del Grado de Maestro de Primaria de la Universidad de Murcia a principios del curso 2014/15. Los errores, en su mayoría, son debidos a un aprendizaje deficiente de hechos, destrezas y conceptos previos. El porcentaje de error supera el 50\% en todos los cursos siendo suspendida la prueba por la mitad de los alumnos. La actitud hacia las Matemáticas es positiva, la ansiedad intermedia y solo la mitad de los alumnos contestó bien la tercera parte de las cuestiones de Matemáticas, lo que nos debe llevar a la creación de programas de mejora en el ámbito de las Matemáticas.
\end{abstract}

Palabras clave: problemas, matemáticas, errores-dificultades, maestro.

\begin{abstract}
This article deals with errors and difficulties which pre-teachers encounter when solving elementary Math problems. The instruments used were: the Maths test which prospective teachers
\end{abstract}

Correspondencia: María Rosa Nortes Martínez Artero (mrosa.nortes@um.es).

Andrés Nortes Checa (anortes@um.es) 
are given when they take the state exams in the Region of Madrid, an attitude questionnaire and an anxiety questionnaire. There were 142 informants, belonging all of them to the Primary School Undergraduate Programme at the University of Murcia at the beginning of the academic year 2014/2015. To a great extent, errors are due to deficient learning of facts, skills and previous concepts. Error rate is over $50 \%$ in all years and half of the students fail the oral test. Their attitude towards Mathematics is positive, there is a medium anxiety level and only half of the students answered the questions about Mathematics correctly, which leads to the conclusion that improvement programmes in the field of Mathematics must be implemented.

Keywords: problems, mathematics, errors-difficulties, teacher.

\section{Introducción}

La resolución de problemas ha sido considerada como eje vertebrador en el Área de Matemáticas en los currículos de enseñanza obligatoria (Ministerio de Educación y Ciencia, 2006) y actualmente "los procesos de resolución de problemas constituyen (...) la piedra angular de la educación matemática” (Ministerio de Educación, Cultura y Deporte, 2014, p. 19386), y al resolver problemas aparecen errores y dificultades en el proceso educativo de los alumnos en todos los niveles.

Los futuros maestros son componentes importantes del proceso educativo y es necesario analizar si cometen errores y si tienen dificultades cuando trabajan las Matemáticas, porque cuando un alumno lleva a cabo un nuevo trabajo y tiene que poner en práctica conocimientos que se supone tiene adquiridos es donde aparecen los errores. Estos son, a veces, tan profundos que cuestionan todo el proceso de enseñanzaaprendizaje de las Matemáticas.

Por otra parte los errores y dificultades en Matemáticas están muy relacionados con la actitud y la ansiedad hacia las mismas, especialmente cuando se resuelven problemas, ya que una serie de dificultades está asociada a las actitudes afectivas y emocionales a las Matemáticas. Socas (2011) indica que el error va a tener procedencias diferentes, pero, en todo caso, va a ser considerado como la presencia en el alumno de un esquema cognitivo inadecuado y no solamente como consecuencia de una falta específica de conocimiento o de un despiste. Legg y Locker (2009) explican que la actitud y ansiedad hacia la Matemática influyen en los procesos metacognitivos del estudiantado y Sachin (2006) establece que estas actitudes pueden ser alimentadas de manera positiva o negativa, según el papel del profesor en las experiencias de aprendizaje, las estrategias de regulación y el manejo de recursos.

\section{Marco teórico}

Los errores y dificultades en la resolución de problemas han sido estudiados desde la vertiente psicopedagógica (Juidias y Rodríguez, 2007), desde la matemática (Rico, 1998; Abrate, Pochulu y Vargas, 2006; Socas, 1997, 2007; Socas, Hernández y Palarea, 2014), desde el Sistema de Numeración Decimal (Salinas, 2007) o en su mayor desarrollo en el álgebra, desde la introducción en primaria hasta su paso a la universidad (Palarea, 1998; Caputo y Macías, 2006; García, 2010; García, Segovia y Lupiáñez, 2011, 2012), entre otros. 
Rico (1998) analiza los errores en el aprendizaje de las Matemáticas y señala que los errores pueden contribuir positivamente en el proceso de enseñanza, que surgen en un marco conceptual consistente basado en conocimientos adquiridos previamente y que, en lugar de culpabilizar a los estudiantes, hay que prevenirlos y que todo proceso de instrucción es potencialmente generador de errores, ya que ellos forman parte de las producciones de los alumnos durante su aprendizaje de las Matemáticas, llegando a preguntarse cuáles son las causas por las que determinados aspectos de un objetivo logran éxitos considerables, mientras que otros presentan dificultades insuperables para los mismos alumnos. Salinas (2007) pone de manifiesto que en la práctica docente, como formadora de maestros, ha constatado lagunas de conocimientos y errores conceptuales en contenidos matemáticos que deberían haberse adquirido y superado en los primeros años de la enseñanza Primaria y llega a asegurar, tras analizar los errores sobre el sistema de numeración decimal cometidos por estudiantes de Magisterio, que los alumnos que inician estos estudios no dominan los contenidos referidos a las Matemáticas escolares, en el sentido de recordar conocimientos adquiridos en las primeras etapas de la enseñanza. Por lo que Socas (2007) ve necesario que, desde la Didáctica de la Matemática, se avance en la delimitación de las causas posibles de los errores que cometen los alumnos para poder actuar, siendo conscientes de las distintas variables que interactúan en el proceso educativo y que condicionan el error al convertirse en dificultades u obstáculos en el aprendizaje. Considera un obstáculo como un conocimiento adquirido, no una falta de conocimiento, que tiene un dominio de eficacia que el alumno lo utiliza en un contexto pero cuando cambia el contexto genera respuestas inadecuadas, incluso incorrectas (Socas, 1997). De ahí que existan errores que tienen su origen en un obstáculo y errores que tienen su origen en ausencia de sentido.

Una de las principales preocupaciones actuales de la Educación Matemática es la investigación en torno a los errores en el proceso de aprendizaje porque, como indican García, Segovia y Lupiáñez (2011) los errores son un tema de constante malestar en los docentes de todos los niveles y su análisis sirve para ayudar al docente a organizar estrategias para una mejor enseñanza/aprendizaje. Y De Castro (2012) distingue entre complejidad, error y dificultad en el proceso de enseñanzaaprendizaje de las Matemáticas, indicando que una tarea es difícil para un alumno si el alumno no tiene el nivel de competencia necesario para resolverla; que la complejidad está en la tarea que hay que resolver y el error es el resultado de la valoración que el profesor emite sobre la producción de un alumno al compararla con la ejecución esperada.

El error es un elemento medular en las propuestas de enseñanza de las Matemáticas constructivistas, pues, como indican Huitrado y Climent (2013), es reconocido como indicador de la comprensión del alumno. Es, por ello, que deben buscarse las causas que lo producen, pudiendo abordarse las dificultades, como indica Socas (2007), desde varias perspectivas: desarrollo cognitivo de los alumnos, currículo de Matemáticas y métodos de enseñanza; el error se considerará como un esquema cognitivo inadecuado y no solo como consecuencia de la falta de conocimiento y Socas et al. (2014) tras aplicar una prueba a futuros profesores de primaria y de secundaria se encuentran con estudiantes que no tienen dificultades para resolverla 
y otros que tienen verdaderas dificultades; entre los primeros destacan una variedad de razonamientos utilizando diferentes tipos de conocimiento y desarrollando procesos como la generalización y la modelización. El origen de las dificultades se encuentra, en general, asociado a la complejidad de los objetos matemáticos implicados en el problema, teniendo que releerlo varias veces e irlo desglosando en partes; a veces tienen dificultad en determinar el orden en el que hay que aplicar las operaciones o en determinar lo que pide el problema; en su mayor parte utilizan el razonamiento de ensayo-error y la analogía.

En ocasiones, los errores y dificultades están asociados a actitudes afectivas y emocionales hacia las Matemáticas, de ahí que la ansiedad y la actitud hacia esta disciplina sea fundamental en los futuros maestros. Aunque los errores se les suelen atribuir solo a los estudiantes, en el proceso educativo intervienen, además de ellos, el profesor, los programas, los planes de estudios, la escuela, las condiciones en que se desarrolla la tarea escolar y los ambientes cultural y social, entre otros.

A veces los errores se cometen al aplicar un algoritmo y prueba de ello es lo manifestado en el boletín de la Sociedad Española de Investigación en Educación Matemática (SEIEM): “Los alumnos que cursan el Grado de Maestro de Primaria, no solo tienen dificultades para resolver problemas elementales, a veces esas dificultades las tienen al aplicar los algoritmos de multiplicación y división" (SEIEM, 2014, p. 2). Por ello, vamos a analizar los errores cometidos al aplicar una prueba de Matemáticas elementales a una muestra de futuros maestros.

\section{Objetivo}

El objetivo es identificar y analizar los errores cometidos por una muestra de alumnos matriculados el curso 2014/15 en el Grado de Maestro de Primaria en Murcia al realizar la Prueba de Matemáticas para el ingreso en el cuerpo de maestros de la Comunidad de Madrid y ver si los resultados obtenidos están relacionados con su dominio afectivo.

\section{Metodología}

\section{Muestra}

La componen 142 estudiantes del Grado de Maestro de Educación Primaria de la Universidad de Murcia, de los que 60 son de $2 .^{\circ}$ curso, 35 de $3 .^{\circ}$ y 42 de $4 .^{\circ}$ matriculados en Matemáticas y su didáctica I (12 créditos en 2. ․ㅡ), Matemáticas y su didáctica II (9 créditos en 3. ํ) y Taller de Matemáticas (3 créditos en $4 .^{\circ}$ ) en el curso 2014/15.

\section{Instrumentos}

1) Prueba de Matemáticas para el ingreso en el Cuerpo de Maestros de Primaria de

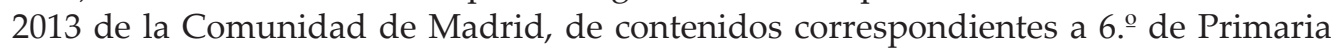
que consta de 15 cuestiones (Comunidad de Madrid, 2013). 
2) Prueba de Actitud hacia las Matemáticas de Auzmendi (1992), cuestionario que se presenta mediante 25 afirmaciones elaboradas para que el encuestado indique hasta que punto está de acuerdo o en desacuerdo con las ideas ahí expresadas (escala Lickert de 1 a 5).

3) Escala de Ansiedad hacia las Matemáticas de Fennema y Sherman (1976) que consta de 12 ítems, cada uno tiene cinco posibles respuestas, siguiendo la escala Lickert.

Las tres pruebas se pasaron a principios del curso 2014/15 para obtener información sobre los conocimientos previos a modo de evaluación inicial.

\section{Resultados}

A continuación se efectúa un estudio cuantitativo de la muestra. Los resultados globales de Actitud hacia las Matemáticas (Auzmendi, 1992) y Ansiedad hacia las Matemáticas (Fennema y Sherman, 1976) tienen una puntuación de 1 a 5 y la Prueba de Matemáticas una puntuación de 0 a 10. Los resultados se recogen en la Tabla 1.

Tabla 1

Resultados representativos de la muestra

\begin{tabular}{lcccc}
\hline Muestra & Edad & Actitud & Ansiedad & Prueba \\
\hline Casos & 142 & 142 & 142 & 142 \\
Media & 22,26 & 3,31 & 2,98 & 3,55 \\
Desv. típica & 5,81 & 0,49 & 0,70 & 1,87 \\
Hombre & 21,85 & 3,35 & 2,63 & 3,97 \\
Mujer & 22,43 & 3,29 & 3,12 & 3,37 \\
p-valor & .597 & .501 & .000 & .084 \\
\hline
\end{tabular}

- La fiabilidad de las escalas de medida se ha efectuado con el coeficiente alfa de Cronbach, obteniendo para Actitud 0,876, para Ansiedad 0,873 y para la Prueba de Matemáticas 0,713.

- Las correlaciones de Pearson Actitud-Ansiedad es de -0,694, entre Actitud-Prueba es de 0,277 y entre Ansiedad-Prueba de -0,353.

Obtenemos los valores de las variables por curso o por género, recogidos en la Tabla 2.

Tabla 2

Resultados por curso o género

\begin{tabular}{|c|c|c|c|c|c|c|}
\hline $\begin{array}{l}\text { Curso o } \\
\text { Género }\end{array}$ & 2.. & $3 .^{\mathrm{o}}$ & 4..$^{\mathrm{o}}$ & Homb. & Muj. & Total \\
\hline Actitud & 3,38 & 3,11 & 3,36 & 3,35 & 3,29 & 3,31 \\
\hline Ansiedad & 2,88 & 3,21 & 2,93 & 2,63 & 3,12 & 2,98 \\
\hline Prueba & 3,17 & 3,45 & 4,11 & 3,97 & 3,37 & 3,55 \\
\hline Casos & 60 & 35 & 47 & 42 & 100 & 142 \\
\hline
\end{tabular}


- En Ansiedad por género hay diferencias significativas al aplicar una t de Student $(p=0,000)$, siendo mayor la ansiedad en mujeres que en hombres.

- En Actitud, por curso hay diferencia significativa al aplicar una F de Snédecor $(\mathrm{p}=0,017)$ a favor de $2 .^{\mathrm{o}}$ y $4 .^{\mathrm{o}}$

- En la Prueba de Matemáticas, por curso hay diferencia significativa al aplicar una F de Snédecor $(p=0,031)$ a favor de 4. .

- La puntuación obtenida para cada una de las cuestiones por sexo, indica que solo es significativa la diferencia de medias a favor de hombres en OP11 $(p=0,024)$ al aplicar una t de Student.

Clasificando por curso y género se obtienen los resultados de la tabla 3.

Tabla 3

Resultados por curso y género

\begin{tabular}{|c|c|c|c|c|c|c|}
\hline $\begin{array}{l}\text { Curso y } \\
\text { Género }\end{array}$ & 2. $\stackrel{\mathrm{o}}{\mathrm{H}}$ & 2. $\stackrel{\mathrm{o}}{\mathrm{M}}$ & 3. ${ }^{\circ} \mathrm{H}$ & 3. $\stackrel{\mathrm{M}}{\mathrm{M}}$ & $4 .^{\mathrm{o}} \mathrm{H}$ & 4..$^{\mathrm{o}} \mathrm{M}$ \\
\hline Actitud & 3,55 & 3,31 & 3,00 & 3,14 & 3,32 & 3,38 \\
\hline Ansiedad & 2,43 & 3,07 & 3,07 & 3,27 & 2,60 & 3,08 \\
\hline Prueba & 4,19 & 2,73 & 3,04 & 3,59 & 4,27 & 4,04 \\
\hline Casos & 18 & 42 & 9 & 26 & 15 & 32 \\
\hline
\end{tabular}

- Clasificados los resultados por género y curso, la actitud más alta la obtienen hombres de $2 .^{\circ}$ con una media de 3,55 y una ansiedad más baja de 2,43 , mientras que en la prueba son hombres de $4 .^{\circ}$ con 4,27 .

- Los peores resultados en actitud son para hombres de 3.ำ con 3,00, ansiedad para mujeres de 3. ${ }^{\circ}$ con 3,27 y en la prueba para mujeres de $2 .^{\circ}$ con 2,73 .

- En 2..$^{\circ}(p=0,001)$ y 4..$^{\circ}(p=0,029)$ las mujeres tiene más ansiedad que los hombres al aplicar una t de Student.

- En 2..$^{\circ}$ los hombres obtienen en la prueba de Matemáticas una diferencia significativa respecto de las mujeres al aplicar la t de Student $(p=0,006)$.

El número de alumnos según cuestiones correctas viene en Tabla 4.

Tabla 4

Alumnos según cuestiones correctas

\begin{tabular}{lccccccccccccc}
\hline N.. Correctas & $\mathbf{0}$ & $\mathbf{1}$ & $\mathbf{2}$ & $\mathbf{3}$ & $\mathbf{4}$ & $\mathbf{5}$ & $\mathbf{6}$ & $\mathbf{7}$ & $\mathbf{8}$ & $\mathbf{9}$ & $\mathbf{1 0}$ & $\mathbf{1 1}$ & $\mathbf{1 3}$ \\
\hline Hombre (42) & 0 & 0 & 4 & 5 & 5 & 5 & 8 & 3 & 5 & 2 & 2 & 2 & 1 \\
Mujer (100) & 2 & 8 & 10 & 12 & 12 & 14 & 12 & 16 & 3 & 1 & 5 & 4 & 1 \\
Total (142) & 2 & 8 & 14 & 17 & 17 & 19 & 20 & 19 & 8 & 3 & 7 & 6 & 2 \\
\hline
\end{tabular}


- Ningún alumno contestó correctamente a su totalidad.

- La mediana en total, hombres y mujeres, es 5.

Las cuestiones analizadas se presentan, a continuación, en tres figuras con los enunciados y en tres tablas en las que cada una de las cuestiones se valoran como bien, en blanco o con error.

\section{OP1.}

a) Halla todos los DIVISORES del número 63.

b) Marca cuáles de los siguientes números son primos: $57-23-77-41-121$.

\section{OP2.}

a) Escribe en números ROMANOS: 1260 - 2013.

b) Escribe en números ARÁBIGOS: MCDLXV MCCCXLIV.

\section{OP3.}

Ordena de MENOR A MAYOR los siguientes números, expresando previamente las fracciones en números decimales: $4 / 5-0,7-2 / 3-0,45-3 / 4$.

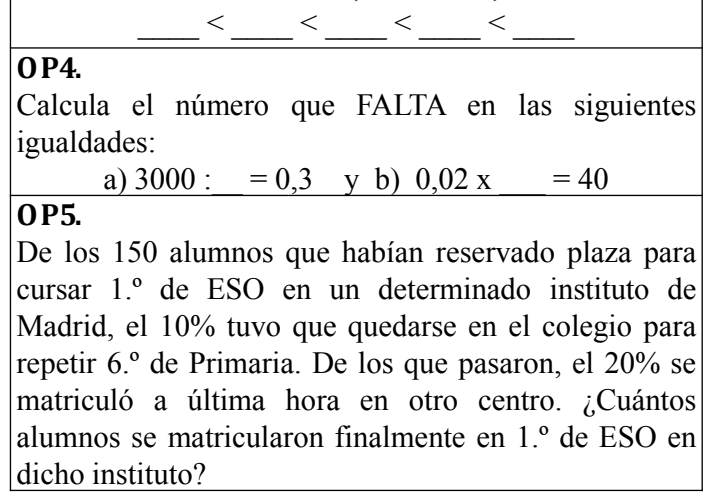

Figura 1. Enunciados $1-5$

Tabla 5

Resultados por cuestión en porcentaje (1)

\begin{tabular}{lcccccccc}
\hline Cuestión & OP1a & OP1b & OP2a & OP2b & OP3 & OP4a & OP4b & OP5 \\
\hline Bien & 10,6 & 17,6 & 38,0 & 57,7 & 69,7 & 66,2 & 58,5 & 50,7 \\
Blanco & 23,9 & 23,2 & 38,7 & 27,5 & 7,0 & 9,9 & 16,2 & 12,0 \\
Error & 65,5 & 59,2 & 23,2 & 14,8 & 23,2 & 23,9 & 25,3 & 37,3 \\
\hline
\end{tabular}

- En OP1a los alumnos que se enfrentan a esta cuestión intentan en su mayoría solo la descomposición del número 63 en producto de factores primos $\left(3^{2} \times 7\right)$, por lo que una parte de ellos indican solo como divisores 3 y 7 . 
- En OP1b el error más cometido es considerar un número compuesto como primo; 57 lo consideran primo 27 alumnos, 121, lo consideran 5 alumnos y 77 lo consideran 4 alumnos.

- En OP2 los errores en esta cuestión están, por una parte debidos a la interpretación incorrecta del lenguaje o en el uso incorrecto de la notación en cuanto al valor de cada símbolo y por otra en cuanto a su colocación.

- En OP3 hay pocos errores, pero los que aparecen son muy graves, como expresar $4 / 5$ como 4,5 ; indicar que $3 / 4$ es 0,3 , que $3 / 4$ es 0,615 o poner $2 / 3$ como 0,89 . Lo que nos indica que el algoritmo de la división no se aplica correctamente.

- En OP4a hay 15 alumnos que dan como respuesta un número decimal inferior a la unidad, mientras que en OP4b el error repetido es dar como resultado 200.

- El OP5 el error más repetido es el de aplicar los dos porcentajes sobre 150, eso lo hacen 12 alumnos de 2. ${ }^{\circ}$, 4 de 3..$^{\circ}$ y 7 de $4 .{ }^{\circ}$, que supone el $43,4 \%$ del total de errores.

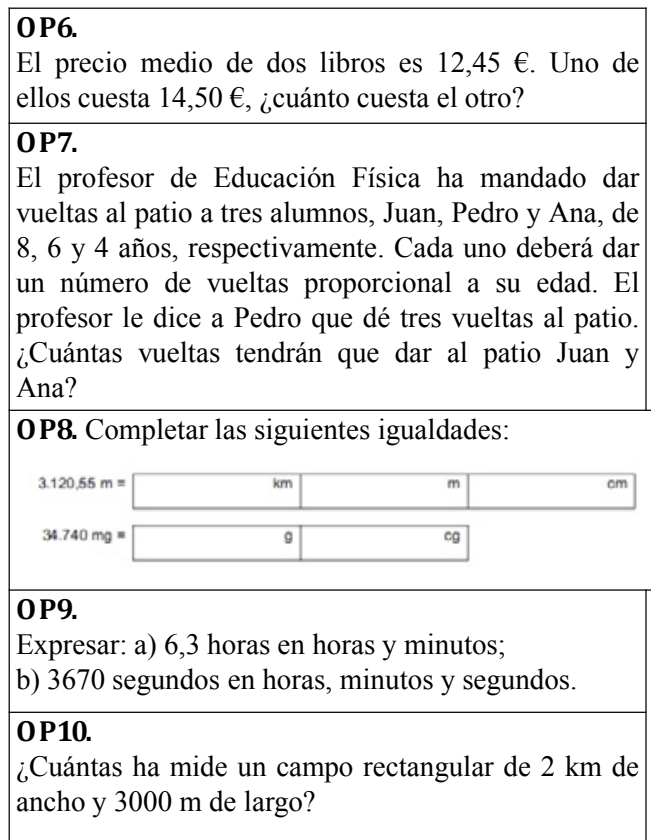

Figura 2. Enunciados 6-10

Tabla 6

Resultados por cuestión en porcentaje (2)

\begin{tabular}{lccccccc}
\hline Cuestión & OP6 & OP7 & OP8a & OP8b & OP9a & OP9b & OP10 \\
\hline Bien & 66,9 & 80,3 & 23,9 & 9,2 & 21,8 & 26,1 & 7,0 \\
Blanco & 21,1 & 12,0 & 9,9 & 12,7 & 22,5 & 37,3 & 57,0 \\
Error & 12,0 & 7,7 & 66,2 & 78,1 & 55,6 & 36,6 & 35,9 \\
\hline
\end{tabular}


- En OP6 el 15,2\% de las respuestas tiene errores matemáticos. Los habituales al tomar los datos equivocados, al traducir el enunciado y otros debidos a cálculos erróneos. Más graves son el desconocimiento de la media o al quitar denominadores.

- En OP7, que ha sido resuelta correctamente por el 80,3\% de los alumnos y dejada en blanco por el $12 \%$, hace que tan solo existan el 7,7\% de errores debido a una interpretación equivocada del enunciado.

- El error cometido en OP8a, mayoritariamente, es el no haber interpretado correctamente los recuadros para expresar una medida de forma incompleja a forma compleja. Tanto es así que en 2. ${ }^{\circ}$ este error lo cometen el $100 \%$, en 3.․ el 92,3\% y en $4 .{ }^{\circ}$ el 70,3\%.

- En OP8b ocurre otro tanto, si bien al tener solo dos recuadros hace que se reduzca el error de interpretación cometiéndolo solo el 36,4\% los alumnos de 2. ․ el 41,4\%

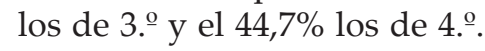

- El error más repetido en el apartado OP9a es traducir 6,3 horas a 6 h 30 min, como si las medidas horarias se expresaran en el sistema de numeración decimal. Esto ha sido del $27,8 \%$ en $2 .^{\circ}$, del $29,4 \%$ en $3 .^{\circ}$ y del $26,9 \%$ en $4 .{ }^{\circ}$.

- En el OP9b se presentan el 36,6\% de los errores en la muestra, siendo un error repetido en 2.o ${ }^{\circ}$ 3. ${ }^{\circ}$ y $4 .^{\circ}$ el efectuar la primera división entre 60 para encontrar los minutos, simplificar y ya al volver a dividir entre 60 para encontrar las horas obtienen como resultado $1 \mathrm{~h} 1 \mathrm{~min} 1 \mathrm{~s}$, siendo el 13,5\% del total de errores.

- En OP10 el desconocimiento de la medida agraria de hectárea (ha) hace que solo el 7\% conteste bien a esta cuestión, encontrándose errores de todo tipo en el cambio de unidad de medida.

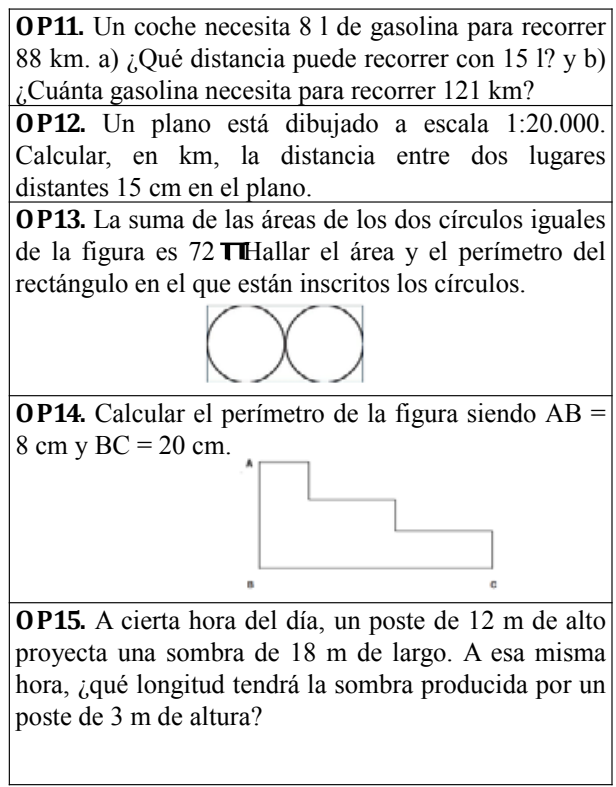

Figura 3. Enunciados 11-15 
Tabla 7

Resultados por cuestión (3)

\begin{tabular}{lcccccc}
\hline Cuestión & OP11a & OP11b & OP12 & OP13 & OP14 & OP15 \\
\hline Bien & 64,8 & 63,4 & 14,1 & 7,0 & 29,6 & 31,0 \\
Blanco & 12,0 & 19,0 & 57,7 & 76,1 & 55,6 & 41,5 \\
Error & 23,2 & 17,6 & 28,2 & 16,9 & 14,8 & 27,5 \\
\hline
\end{tabular}

- En OP11 solamente el $26,4 \%$ de los que responden comete error en la primera parte, al calcular la distancia que puede recorrer con 151 , y el 21,7\% en la segunda parte, al calcular cuánta gasolina necesita para recorrer $121 \mathrm{~km}$, siendo los errores cometidos mayoritariamente en la aplicación de los algoritmos y en no revisar la solución obtenida. Eso hace que se den respuestas como que con 15 litros se pueden recorrer $1320 \mathrm{~km}$ y que para recorrer $121 \mathrm{~km}$ se necesitan 1,3 litros.

- En OP12 el 20\% de los errores cometidos es por no saber aplicar una escala, el $32,5 \%$ al pasar de unas unidades a otras y otro $25 \%$ da respuestas incoherentes como que la distancia entre dos lugares que distan $15 \mathrm{~cm}$ en el plano es de $300.000 \mathrm{~km}$.

- En OP13 lo más significativo es la gran cantidad de alumnos que lo deja en blanco, el $86,7 \%$ en 2. ${ }^{\circ}$, el $74,3 \%$ en 3. ${ }^{\circ}$ y el $63,8 \%$ en $4 .^{\circ}$ De los alumnos que cometen error, el 20,8\% confunden la longitud de la circunferencia con el área del círculo y el 8,3\% contesta a otra cosa distinta.

- En OP14 este problema lo dejan en blanco el 55,6\% de los alumnos de la muestra. De los que responden, tienen errores de estimación de las medidas no dadas, a partir del dibujo, el $20 \%$ en $2 .{ }^{\circ}$, el $25 \%$ en $3 .^{\circ}$ y el $21,4 \%$ en $4 .{ }^{\circ}$.

- En OP15 de los errores cometidos el 43,6\% corresponde al aplicar el algoritmo de la división y el 12,8\% que aplican el teorema de Pitágoras no se sabe para qué. Descontando los alumnos que lo dejan en blanco, el 61,5\% comete errores

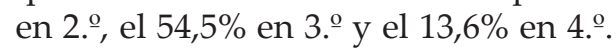

De todas las cuestiones se ha obtenido el porcentaje de error considerando las cuestiones respondidas, representándolos en las tablas 8, 9 y 10 en donde se sombrean los casos superiores al 50\%.

Tabla 8

Porcentajes de error por curso y cuestión (1)

\begin{tabular}{ccccccccc}
\hline Curso & OP1a & OP1b & OP2a & OP2b & OP3 & OP4a & OP4b & OP5 \\
\hline 2.$^{\text {. }}$ & 90,7 & 80,9 & 51,6 & 15,4 & 51,4 & 30,0 & 34,0 & 48,1 \\
. $^{\text {- }}$ & 88,5 & 64,0 & 27,3 & 20,8 & 25,0 & 35,5 & 32,1 & 37,9 \\
. $^{-}$ & 79,5 & 81,1 & 32,4 & 25,0 & 14,0 & 17,0 & 25,0 & 38,6 \\
Total & 86,1 & 77,1 & 37,9 & 22,6 & 25 & 26,6 & 30,3 & 42,4 \\
\hline
\end{tabular}


Tabla 9

Porcentajes de error por curso y cuestión (2)

\begin{tabular}{cccccccc}
\hline Curso & OP6 & OP7 & OP8a & OP8b & OP9a & OP9b & OP10 \\
\hline 2. $^{\text {o }}$ & 18,2 & 7,4 & 59,6 & 86,3 & 81,4 & 64,7 & 84,6 \\
3. $^{\text {- }}$ & 14,8 & 10,3 & 83,9 & 93,5 & 63,0 & 76,2 & 86,7 \\
4. $^{\text {- }}$ & 12,2 & 9,5 & 82,2 & 90,5 & 66,7 & 41,2 & 80,0 \\
Total & 15,2 & 8,8 & 73,4 & 89,5 & 71,8 & 58,4 & 83,6 \\
\hline
\end{tabular}

Tabla 10

Porcentajes de error por curso y cuestión (3)

\begin{tabular}{cccccccc}
\hline Curso & OP11a & OP11b & OP12 & OP13 & OP14 & OP15 & Total \\
\hline $\mathbf{2 .}^{\mathbf{o}}$ & 33,3 & 24,5 & 57,9 & 75,0 & 46,7 & 61,5 & $\mathbf{4 9 , 9}$ \\
$\mathbf{3 .}^{\mathbf{o}}$ & 22,6 & 22,6 & 80,0 & 88,9 & 25,0 & 54,5 & $\mathbf{5 2 , 3}$ \\
$\mathbf{4 .}^{\mathbf{o}}$ & 20,0 & 17,1 & 61,9 & 58,8 & 17,6 & 13,6 & $\mathbf{4 4 , 1}$ \\
Total & 26,4 & 21,7 & 66,7 & 70,6 & 33,3 & 47,0 & $\mathbf{4 8 , 5}$ \\
\hline
\end{tabular}

- Las cuestiones OP1, OP8, OP10, OP12 y OP13, tiene un porcentaje de error superior al $50 \%$ en los tres cursos y en OP9 y OP15 en 2.ํ y 3.․

\section{Discusión}

La resolución de un problema provoca en algunos alumnos una actitud negativa acompañada de una falta de confianza. La dificultad con la que se encuentra un alumno hace que aumente la ansiedad y obtenga peores resultados. En el estudio realizado, si bien la actitud y la ansiedad están equilibradas, los resultados de Matemáticas son bajos, la correlación entre actitud y ansiedad es alta y negativa $(-0,694)$, la ansiedad y la prueba tienen una correlación baja y negativa $(-0,353)$ y que la actitud y prueba tienen una correlación baja y positiva $(0,277)$, los datos indican que una actitud positiva hacia las matemáticas $(3,31)$ puede llevar a buenos resultados, pero no necesariamente.

El hecho de que un alumno puntúe el ítem más alto "Me provoca una gran satisfacción el llegar a resolver problemas de matemáticas" $(4,49)$, más en mujeres $(4,54)$ que en hombres $(4,38)$, denota la importancia de tener una actitud favorable hacia las matemáticas. Por el contrario, el estar de acuerdo con "Casi siempre me pongo nervioso en un examen de matemáticas" $(3,82)$, más en mujeres $(4,03)$ que en hombres $(3,33)$ denota que esta situación es un factor añadido a la hora de cometer errores debido a la falta de seguridad y serenidad de actuación. Corrobora lo indicado por Socas (1997) de que hay unas dificultades no solamente asociadas a los procesos cognitivos de los alumnos, a los procesos de enseñanza, a los procesos del pensamiento matemático, a la complejidad de los objetos matemáticos, sino también asociadas a actitudes afectivas y emocionales de las Matemáticas. 
Que el 28,24\% de las cuestiones quedaran sin contestar y que de las que contestaron en cinco de ellas (OP1, OP8, OP10, OP12 y OP13) el porcentaje de errores supere

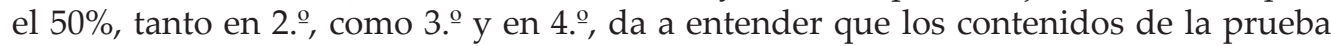
correspondientes a Primaria no se tienen adquiridos, lo que indica también la puntuación media de la muestra que es 3,55.

La mayoría de los errores cometidos por los alumnos se pueden considerar como errores debidos a un aprendizaje deficiente de hechos, destrezas y conceptos previos (Radatz, 1980) y como datos mal utilizados, falta de verificación de la solución y errores de cálculo (Movshovitz-Hadar, Zaslavsky e Inbar, 1987).

Los errores mencionados se encuentran dentro de las dificultades encontradas por Socas et al. (2014) que citaban falta de comprensión del texto, no saber el significado de las palabras, no comprender globalmente el texto, errores en operaciones, algoritmos y técnicas, falta de conocimientos de los procesos, de los razonamientos, entre otras.

De ahí que, como indican Mato, Espiñeira y Chao (2014), los profesionales de la enseñanza debamos centrar nuestra atención cada vez más en las barreras a las que se enfrentan los estudiantes mientras aprenden, y proponen considerar el dominio afectivo en los planes de estudio de los maestros a la par que se trabaja el componente cognitivo.

La Geometría como dicen Abrate et al. (2006) demanda una notación particular y definiciones muy específicas, mucho más de lo que requiere la Aritmética y el Álgebra y, en muchos casos, es porque el alumno no posee elementos para decodificar el mensaje que se le presenta. Y esto pasa en la cuestión OP13 en la que aparecen dos circunferencias tangentes inscritas en un rectángulo que solo es contestada bien por el 7\% y tres de cada cuatro alumnos la deja en blanco. La interpretación incorrecta del dibujo y la confusión entre longitud de la circunferencia y área del círculo viene a reafirmar la confusión entre perímetro y área de una figura geométrica (Nortes y Nortes, 2013). Pero también pasa en la cuestión siguiente (OP14) en la que hay dibujado un polígono en forma de escalera con unos datos y se pide calcular su perímetro, porque más de la mitad de los alumnos la deja en blanco, siendo el error más cometido el obtener del dibujo unos datos por intuición que el enunciado no indica, porque cuando un alumno se encuentra ante un problema que no sabe por donde intentar resolverlo puede caer en el riesgo de no saber por qué hace algo o para qué le va a servir. Una muestra de esto es el último problema (OP15), en el que uno de cada ocho alumnos aplica indebidamente el teorema de Pitágoras y dos de cada cinco hacen mal una división elemental.

\section{Conclusiones}

La preocupación por el bajo rendimiento en el área de Matemáticas promueve la búsqueda de nuevas fórmulas de actuación docente (García-García, Biencinto-López, Carpintero-Molina, Núñez-del-Río y Arteaga-Martínez, 2013) y una de ellas es aprender de los errores. Muchos de los errores que hemos mencionado son debidos a que los alumnos aprendieron una serie de mecanismos para resolver problemas, unas rutinas y recetas, pero no llegaron a adquirir un razonamiento profundo, derivando en las dificultades con las que se encuentran para resolver problemas elementales 
de Matemáticas. Orton (1990) indica que una considerable parte de alumnos cuando deja la enseñanza obligatoria no ha alcanzado la etapa de las operaciones formales, ignorando si lo consigue alguna vez. Y, en este caso, creemos que muchos alumnos se han quedado en la etapa de las operaciones concretas y les resulta casi imposible tener acceso a las abstracciones. Socas et al. (2014) mencionan que los estudiantes para profesor tienen dificultad para identificar con claridad lo que le piden o para aplicar la heurística, predominando el ensayo-error y la analogía para resolver problemas.

Cuando un alumno no entiende, no le gustan las Matemáticas o no sabe para qué sirve lo que está haciendo, lo que intenta es buscar respuestas rápidas para solucionar los problemas y demanda fórmulas o procedimientos a modo de recetas ya que, como dice Abrate et al. (2006), de esta forma puede obtener resultados rápidos, pero con mayor dependencia de su memoria y sus producciones se vuelven más vulnerables a la aparición de errores al no encontrar un mecanismo que le permita verificar si lo que hace es correcto.

Se ha constatado el alto porcentaje de errores en la muestra, que de cada dos respuestas una es con error $(46,2 \%)$ y que el error se mantiene a lo largo de los cursos pese a haber cursado los alumnos créditos de Matemáticas y su didáctica, lo que nos viene a decir que las dificultades en Matemáticas elementales con las que llegan los alumnos a los estudios del Grado de Maestro las siguen manteniendo, los errores básicos no disminuyen, y las lagunas de conocimientos, como indica Salinas (2007), no se rellenan, lo que hace que nos tengamos que replantear la labor de formadores de futuros maestros. Y puesto que los futuros maestros optan por estilos activos de enseñanza (González y Pino, 2014) habrá que diseñar programas más eficaces de formación inicial del profesorado, ya que en ocasiones el profesorado encargado de formar a futuros maestros "ha de enseñar a sus estudiantes contenidos elementales propios de la etapa educativa para la que éstos se están preparando como maestros como si fuera la primera vez que estos estudiantes los abordan" (SEIEM 2014, p. 2). Que el $54,2 \%$ de los alumnos $\left(63,3 \%\right.$ de $2 . .^{\circ}, 60 \%$ de $3 .^{\circ}$ y $38,3 \%$ de $4 .^{\circ}$ ) contestara bien a cinco o menos cuestiones y los numerosos errores encontrados en la presente investigación son motivo de reflexión para crear programas de mejora en los estudios del Grado de Maestro de Educación Primaria en el ámbito de las Matemáticas.

\section{Bibliografía}

Auzmendi, E. (1992). Las actitudes hacia las matemáticas-estadística en las enseñanzas medias y universitaria. Bilbao: Mensajero.

Abrate, R., Pochulu, M. y Vargas, J. (2006). Errores y dificultades en Matemáticas. Argentina: Universidad Nacional de Villa María. Recuperado de http://unvm.galeon.com/ Libro1.pdf

Comunidad de Madrid (2013). Procedimiento selectivo para el ingreso en el cuerpo de maestros: matemáticas. Recuperado de http://sec.magister.com.es/madrid2013/ resumen_comun_madrid_2013.pdf

Caputo, S. y Macias, D. (2006). Análisis de los alumnos de la asignatura "álgebra I" al elaborar demostraciones. Argentina: Universidad Nacional del Nordeste. Recuperado de http//www.unne.edu.ar/Web/cyt/cyt2006/09-Educacion/ 2006-D-012.pdf. 
De Castro, C. (2012). Estimación en cálculo con números decimales: dificultad de las tareas y análisis de estrategias y errores con maestros en formación. (Tesis Doctoral). Recuperada del Repositorio Institucional de la Universidad de Granada.

Fennema, E. y Sherman, J.A. (1976). Fennema-Sherman mathematics attitude scales. Instruments designed to measure attitudes toward the learning of mathematics by males and females. JSAS Catalog of Selected Documents of Psychology, 6(31).

García, J. (2010). Análisis de errores y dificultades en la resolución de tareas algebraicas por alumnos de primer ingreso en el nivel licenciatura (Trabajo Fin de Máster inédito). Universidad de Granada, Granada.

García, J., Segovia, I. y Lupiáñez, J.L. (2011). Errores y dificultades de estudiantes mexicanos de primer curso universitario en la resolución de tareas algebraicas. En J. L. Lupiáñez, M.C. Cañadas, M. Molina, M. Palarea y A. Maz (Eds.), Investigaciones en Pensamiento Numérico y Algebraico e Historia de la Matemática y Educación Matemática 2011 (pp. 145-155). Granada: Universidad de Granada.

García, J., Segovia, I. y Lupiáñez, J.L. (2012). Antecedentes y fundamentación de una investigación sobre errores en la resolución de tareas algebraicas. En D. Arnau, J. L. Lupiáñez y A. Maz (Eds.), Investigaciones en Pensamiento Numérico y Algebraico e Historia de la Matemática y Educación Matemática 2012 (pp. 139-148). Valencia: Departamento Didáctica de la Matemática de Valencia y SEIEM.

García-García, M., Biencinto-López, C. Carpintero-Molina, E., Núñez-del-Río, M.C. y Arteaga-Martínez, B. (2013). Rendimiento en Matemáticas y actitud hacia la materia en centros inclusivos: estudio en la Comunidad de Madrid. Revista de Investigación Educativa, 31(1), 117-132.

González, M. y Pino, M. (2014). Aproximación a las representaciones y creencias del alumnado de Magisterio sobre los estilos de enseñanza. Educación XXI, 17(1), 83-100.

Huitrado, J. L. y Climent, N. (2013). Conocimiento profesional del profesor ante errores relativos al álgebra de los alumnos de secundaria. En A. Berciano, G. Gutiérrez, A. Estepa y N. Climent (Eds.), Investigación en Educación Matemática XVII (pp. 327-336). Bilbao: SEIEM.

Juidias, J. y Rodríguez, I. (2007). Dificultades de aprendizaje e intervención psicopedagógica en la resolución de problemas matemáticos. Revista de Educación, 342, 257-286.

Legg, A. y Locker, L. (2009). Math performance and its relationship to math anxiety and metacognition. North American Journal of Psychology. 11(3). Recuperado de http:// digitalcommons.georgiasouthern.edu/cgi/viewcontent.cgi?article=1428\&context=etd.

Mato, D., Espiñeira, E. y Chao, R. (2014). Dimensión afectiva hacia la matemática: resultados de un análisis en educación primaria. Revista de Investigación Educativa, 32 (1), 57-72.

Ministerio de Educación y Ciencia (2006). Real Decreto 1513/2006 de 7 de diciembre, por el que se establecen las enseñanzas mínimas en Educación Primaria. BOE 293 de 8 de diciembre de 2006.

Ministerio de Educación, Cultura y Deporte (2014). Real Decreto 126/2014, de 28 de febrero, por el que se establece el currículo básico de la Educación Primaria. BOE 52 de 1 de marzo de 2014.

Movshovitz-Hadar, N.; Zaslavsky, O. e Inbar, S. (1987). An empirical classification model for errors in high school mathematics. Journal for Research in Mathematics Education, 18(1), 3-14. 
Nortes, R. y Nortes, A. (2013). Perímetro y Área. Un problema en futuros maestros. Números, 84, 65-85

Orton, A. (1990). Didáctica de las Matemáticas. Madrid: MEC-Morata.

Palarea, M.M. (1998). La adquisición del Lenguaje Algebraico y la detección de errores comunes cometidos en Álgebra por alumnos de 12 a 14 años. (Tesis Doctoral inédita). Universidad de La Laguna, Tenerife.

Radatz, H. (1980). Student's Errors in the Mathematics Learning Process: A Survey. For the Learning of Mathematics, 1(1), 16-20.

Rico, L. (1998). Errores en el aprendizaje de las matemáticas. En J. Kilpatrick, L. Rico y P. González (Eds.). Educación matemática, 69-109. México: Grupo Editorial Iberoamericana.

Sachin, J. (2006). Mathematics and test anxiety as a function of mediated learning experience and metacognitive skills. Recuperado de http://proquest.umi.com/pqdlink?did=1232 418141 yFmt=7yclientId=79356yRQT=309yVName=PQD

Salinas, M.J. (2007). Errores sobre el sistema de numeración decimal en estudiantes de Magisterio. En M. Camacho, P. Flores y P. Bolea (Eds.), Investigación en Educación Matemática XI (pp. 381-390). La Laguna: SEIEM.

Sociedad Española de Investigación en Educación Matemática (2014). Editorial. Boletín, $37,2$.

Socas, M.M. (1997). Dificultades, obstáculos y errores en el aprendizaje de las matemáticas en la educación secundaria. En Rico, L. (Ed.), La educación matemática en la enseñanza secundaria (pp. 125- 152). Barcelona: Horsori.

Socas, M.M. (2007). Dificultades y errores en al aprendizaje de las Matemáticas. Análisis desde el enfoque lógico semiótico. En M. Camacho, P. Flores y P. Bolea (Eds.), Investigación en Educación Matemática XI (pp. 19-52). La Laguna: SEIEM.

Socas, M.M. (2011). Aprendizaje y enseñanza de las Matemáticas en Educación Primaria. Buenas prácticas. Educatio Siglo XXI, 29(2), 199-224.

Socas, M.M., Hernández, J. y Palarea, M.M. (2014). Dificultades en la resolución de problemas matemáticos para profesores de educación primaria y secundaria. En J. L. González, J.A. Fernández-Plaza, E. Castro-Rodríguez, M. T. Sánchez-Compaña, C. Fernández, J.L. Lupiáñez y L. Puig (Eds.), Investigaciones en Pensamiento Numérico y Algebraico e Historia de la Matemática y Educación Matemática 2014 (pp. 145-154). Málaga: Dpto. de Didáctica de las Matemáticas, de las Ciencias Sociales y de las Ciencias Experimentales y SEIEM. Recuperado de www.seiem.es/gruposdetrabajo/ pna/ActasPNA2014.pdf

Fecha de recepción: 11/06/2015

Fecha de revisión: 13/06/2015

Fecha de aceptación: 26/10/2015 
